\title{
Enterprise Architecture Planning: Analyses of Requirements from Practice and Research
}

\author{
Emmanuel Nowakowski, Matthias Farwick, Thomas Trojer, Martin Häusler, Johannes Kessler, Ruth Breu \\ University of Innsbruck, Institute of Computer Science, Austria \\ [firstname].[lastname]@uibk.ac.at
}

\begin{abstract}
Enterprise architecture management (EAM) has become an increasingly important topic in practice due to the growing complexity of organizations and their underlying IT. While there is a strong interest in Enterprise Architecture (EA) modeling, evaluation, and frameworks, a lack of knowledge remains in the research field of EA planning. We conducted a series of expert interviews on the topic of EA planning. From these interviews we were able to extract requirements for EA planning from practice as the foundation of our analyses. Additionally, we conducted a structured literature review to elicit requirements for EA planning from a research perspective. This paper combines the results of both the practitioner interviews and the literature review to emphasize the gaps between the two worlds. As a result, we identified that current research does not adequately address the pressing problems of EA planning in practice.
\end{abstract}

\section{Introduction}

EAM is an IT-management discipline that aims at reducing IT-costs and optimizing IT support for business execution. EAM's promise is that by analyzing the dependencies of the current EA, from the business down to the supporting IT-architecture, long-term planning and less risky transformations can be achieved [12,21].

Architecture planning is one of the core EA processes as described in EA frameworks like TOGAF [24]. Its goal is to plan architectural transformations aligned with the strategy of an organization as a whole. This specifically includes transforming IT to meet the (emerging) requirements of the business [15,36].

EA planning is a process in which multiple to-be architectures are constructed to transform the current architecture to a specific target architecture. It includes the modeling of different architectural scenarios and comparing them against each other. A typical example for different scenarios is the problem of redundant ERP systems after a merger. In such a case, the question arises which information system to eliminate. The two scenarios are modeled and compared with each other based on metrics like cost or technical soundness to decide on the best solution.

The current architecture, or as-is architecture, can be seen as a snapshot of the current EA. The to-be models, which are created during an EA planning process, model the steps which are needed to transform the current to the target architecture, where the target architecture realizes the strategic goal of an organization. In Section 3.2 we describe the EA planning process in more detail.

Organizations typically adopt EA frameworks [33] that guide their EA initiatives. However, regarding architecture planning, EA frameworks stay vague on how to actually conduct it [1]. This lack of practical planning guidance is also reflected by the struggles that were reported to us by our interview partners. They include the difficulty to keep visualizations up-to-date since that is time consuming.

As a first step of analyzing the issues concerning EA planning, we conducted interviews with 11 EA practitioners and executed a structured literature review on EA planning. From the interviews we aimed to establish a model on how EA planning is understood and used in practice. The following research questions were asked: RQ1: "What is the common understanding of EA planning among EA practitioners?" (Subsection 3.1), RQ2: "What are the requirements from practice for EA planning?” (Subsection 3.1), and RQ3: “What is the level of abstraction on which EA is planned in practice?" (Subsection 3.1).

Based on the findings from the interviews we started the literature review to explore the body of knowledge on EA planning in research literature (RQ4): "What is the current state of research on EA planning?" and (RQ5): "What requirements are identified and tackled in literature?” (Subsection 3.2). 
Finally, we analyzed the research gap (RQ6): "What is the gap between the issues of EA planning in practice and research?". This question is discussed in Section 4. From this gap analysis we derive our future research agenda around EA planning that we present in Section 5 .

\section{Research Method}

Our work is based on a design science research (DSR) approach. In design science, IT artifacts, which are intended to solve identified organizational problems, are created and evaluated [26]. For that purpose, Hevner [25] proposed a three-cycle approach for conducting DSR, comprising a rigor cycle, a design cycle, and a relevance cycle.

The rigor cycle is connected to the knowledge base of experience, scientific foundations, and expertise that informs the research project [25]. Therefore, the work presented in this paper belongs to the rigor cycle of our research on EA planning. Here, the knowledge base is identified and the relevance of the research topic is tested against it by checking if there exist gaps between the expertise of experts in the field and the research which presents the scientific theories and methods. The design cycle comprises the implementation of our future research results into an EA tool which resulted from our previous work. Additionally, in the relevance cycle the results from the previous cycle are field tested by our industry partners.

Relevance of our research is established by the input from our interview partners which is presented in Subsection 3.1. To establish the knowledge base, we present a structured literature review (SLR) in Subsection 3.2.

In the following section, this data collection is described in more detail including an analysis of the results of the structured literature review and the interviews.

\section{Data Collection}

The data collection is comprised of expert interviews and a structured literature review. They were conducted so that the gaps between research and practice could be identified and relevance for our future work in this area established. Table 1 shows the requirements which resulted from RQ2 and RQ5. The requirement categories, identified in practice (Subsection 3.1) and in research (Subsection 3.2), and the count in how many publications they were mentioned, are shown in the left column. The count of interviewees, who mentioned the specific requirement, is listed in the right column above the participants. Each count is depicted with its percentage value according to the amount of found literature (32) and the number of interviewees (11). Furthermore, each top-level requirement is divided into subcategories and also marked with the requirement types: method/process, tooling, visualization, and data model. The middle column lists the references that mention a specific requirement. Additionally, the right column shows who of the interviewees mentioned the requirement category. Finally, the table is sorted according to the order of appearance in the example, which is to be found in Subsection 3.2, to ensure consistency.

Table 1. EA planning requirements from research literature and expert interviews

\begin{tabular}{|c|c|c|}
\hline Requirement Categories & Listed in Reference & $\begin{array}{l}\text { Mentioned by } \\
\text { Participant }\end{array}$ \\
\hline R1) Ability to plan based on current architecture & $11(\sim 34 \%)$ & $\begin{array}{c}6(\sim 55 \%) \\
\text { P2, P3, P4, P5, P9, } \\
\text { P10 }\end{array}$ \\
\hline $\begin{array}{l}\text { a) ability to analyze dependencies and relationships of as- } \\
\text { is models (method/process) }\end{array}$ & {$[3,4,7]$} & \\
\hline b) as-is model as basis for planning (method/process) & {$[8,19,21,28,33,38,39,43]$} & \\
\hline R2) Ability to evaluate to-be and current architecture & $26(-81 \%)$ & $\begin{array}{c}10(\sim 91 \%) \\
\mathrm{P} 1, \mathrm{P} 2, \mathrm{P} 3, \mathrm{P} 4, \mathrm{P} 5, \\
\mathrm{P} 6, \mathrm{P} 7, \mathrm{P} 9, \mathrm{P} 10, \mathrm{P} 11\end{array}$ \\
\hline a) analyze models (method/process) & $\begin{array}{c}3,4,5,6,7,11,12,22,23,24 \\
31,39,40,41,42,44,46]\end{array}$ & \\
\hline b) compare models (method/process) & $\begin{array}{c}{[3,4,5,6,7,11,15,19,22,24} \\
40,41,44,45]\end{array}$ & \\
\hline c) qualitative and quantitative metrics (method/process) & {$[2,8,10,20,28,31,34,39]$} & \\
\hline R3) Ability to plan different scenarios & $21(\sim 66 \%)$ & $\begin{array}{c}10(\sim 91 \%) \\
\mathrm{P} 1, \mathrm{P} 2, \mathrm{P} 3, \mathrm{P} 4, \mathrm{P} 5, \\
\mathrm{P} 6, \mathrm{P} 7, \mathrm{P} 9, \mathrm{P} 10, \mathrm{P} 11\end{array}$ \\
\hline a) multiple to-be models (method/process) & $\begin{array}{c}2,3,4,5,6,7,8,10,11,13,14 \\
, 20,22,23,34,38,41,43]\end{array}$ & \\
\hline
\end{tabular}




\begin{tabular}{|c|c|c|}
\hline b) different transformation paths (method/process) & {$[18,19,33]$} & \\
\hline R4) Ability to set up transformation paths & $21(\sim 66 \%)$ & $0(0 \%)$ \\
\hline a) transformation model (data model) & {$[3,5,6,15,18]$} & \\
\hline b) derive project timeline (method/process) & {$[3,5,6,18,33,42]$} & \\
\hline $\begin{array}{l}\text { c) derive transformation projects out of to-be models } \\
\text { (method/process) }\end{array}$ & {$[4,7,14,43]$} & \\
\hline d) gap analysis (method/process) & $\begin{array}{c}{[2,7,19,20,22,23,24,29,32} \\
, 34,39,40]\end{array}$ & \\
\hline R5) Ability to define scope of plan & $2(\sim 6 \%)$ & $\begin{array}{l}1(\sim 9 \%) \\
\text { P9 }\end{array}$ \\
\hline a) defined scope in project plan (method/process) & [40] & \\
\hline b) narrow scope for transformation path (method/process) & [33] & \\
\hline R6) Ability to manage architecture management life-cycle & $12(\sim 38 \%)$ & $\begin{array}{c}\text { 7( 64\%) } \\
\text { P2, P3, P4, P6, P7, } \\
\text { P9, P10, }\end{array}$ \\
\hline a) capture individual life-cycles (method/process) & $\begin{array}{c}{[2,3,4,5,6,7,12,16,23,24} \\
32,44]\end{array}$ & \\
\hline b) color-code life-cycle information (visualization) & [14] & \\
\hline R7) Ability to react on unplanned changes & $3(\sim 9 \%)$ & $0(0 \%)$ \\
\hline $\begin{array}{l}\text { a) changes in transformation plans that have an effect on } \\
\text { future to-be models need to be covered (meth- } \\
\text { od/process) }\end{array}$ & {$[7,22]$} & \\
\hline $\begin{array}{l}\text { b) transformation model which captures relevant aspects of } \\
\text { planning and taking dynamic changes into account (data } \\
\text { model) }\end{array}$ & [18] & \\
\hline R8) Availability of an action repository & $3(\sim 9 \%)$ & $0(0 \%)$ \\
\hline a) formal description of actions (method/process) & [18] & \\
\hline $\begin{array}{l}\text { b) action repository for modeled abstract actions (data } \\
\text { model) }\end{array}$ & {$[19,33]$} & \\
\hline R9) Specific visualizations for EA planning & $14(\sim 44 \%)$ & $\begin{array}{c}10(\sim 91 \%) \\
\mathrm{P} 1, \mathrm{P} 2, \mathrm{P} 3, \mathrm{P} 4, \mathrm{P} 5, \\
\mathrm{P} 6, \mathrm{P} 7, \mathrm{P} 9, \mathrm{P} 10, \mathrm{P} 11\end{array}$ \\
\hline a) visualizations for different stakeholders (visualization) & $\begin{array}{c}{[2,4,7,10,11,12,15,21,23} \\
24,31,32,39] \\
\end{array}$ & \\
\hline b) interactive visualizations (visualization) & [30] & \\
\hline $\begin{array}{l}\text { c) view multiple perspectives of the whole and its pieces } \\
\text { (method/process) }\end{array}$ & {$[2,44]$} & \\
\hline R10) Ability to capture historic information & $4(-13 \%)$ & $0(0 \%)$ \\
\hline a) traceability of architecture decisions (method/process) & [13] & \\
\hline b) versioning (method/process) & {$[5,11,38]$} & \\
\hline R11) Availability of up-to-date data and documentation & $7(\sim 22 \%)$ & $\begin{array}{c}3(\sim 27 \%) \\
\text { P6, P10, P11 }\end{array}$ \\
\hline a) up-to-date data and models (data model) & {$[2,10,11,22,23,32,38]$} & \\
\hline b) automated EA documentation (tooling) & [11] & \\
\hline c) high enough data quality (data model) & [22] & \\
\hline R12) Availability of tool support & $4(-13 \%)$ & $\begin{array}{c}3(\sim 27 \%) \\
\text { P3, P4, P7 }\end{array}$ \\
\hline a) ease-of-use (tooling) & {$[2,5,11,22]$} & \\
\hline R13) Ability to freely model & $0(0 \%)$ & $\begin{array}{c}3(\sim 27 \%) \\
\mathrm{P} 1, \mathrm{P} 9, \mathrm{P} 11 \\
\end{array}$ \\
\hline R14) Automated trigger for planning processes & $0(0 \%)$ & $\begin{array}{l}2(\sim 18 \%) \\
\text { P2, P7 }\end{array}$ \\
\hline R15) Automated import from heterogeneous data-sources & $0(0 \%)$ & $\begin{array}{c}2(\sim 18 \%) \\
\text { P1, P4 }\end{array}$ \\
\hline R16) Central model repository for plans & $0(0 \%)$ & $\begin{array}{l}2(\sim 18 \%) \\
\text { P5, P10 }\end{array}$ \\
\hline
\end{tabular}




\subsection{Interviews}

The goal of our interview series was to find out how EA planning is performed in practice. In detail, the common understanding of EA planning among EA practitioners (RQ1), the requirements from practice for EA planning (RQ2), and the abstraction level of planning (RQ3) are extracted. Additionally, the interviews are used to establish the practical relevance for our research.

The interviews were conducted with experts in the field of EAM. Additionally, two IT architects participated in the interviews so that the architects' technical requirements on EA planning could be included. In the time span between February and May 2016, 11 interviews were conducted with interviewees from 10 different enterprises and 8 different industry sectors, in Germany and Austria (see Table 2).

The interviewees were identified from our existing network of industry partners and via XING - a business social network for German-speaking countries. In this network, managing positions in EAM and IT architecture were searched. Nine interviews were conducted face-to-face and two via video conferencing.

An overview of the interviewees' positions and their industry sector can be seen in Table 2. Additionally, the maturity of their organization regarding EAM is shown to properly weight our results. The maturity was assessed by the years of EA experience of the organization and was self-identified by the according interviewee. The interviewees stated that their organizations have problems with the maturity of EAM, especially in the EA planning sector. Because of the diversity in EA maturity among the participants, it is important to interpret the individual answers in the context of their maturity.

Table 2. Interviewee Overview

\begin{tabular}{|c|c|c|c|}
\hline Participant & $\begin{array}{c}\text { EAM } \\
\text { Maturity }\end{array}$ & Role & $\begin{array}{c}\text { Industry } \\
\text { Sector }\end{array}$ \\
\hline P1 & low & EAM & Banking \\
\hline P2 & low & $\begin{array}{c}\text { IT } \\
\text { architect }\end{array}$ & $\begin{array}{c}\text { IT service } \\
\text { provider }\end{array}$ \\
\hline P3 & high & EAM & Semiconductor \\
\hline P4 & medium & EAM & Insurance \\
\hline P5 & high & EAM & Banking \\
\hline P6 & medium & EAM & Pharma \\
\hline P7 & high & EAM & Insurance \\
\hline P8 & medium & EAM & E-Commerce \\
\hline P9 & medium & EAM & $\begin{array}{c}\text { IT service } \\
\text { provider }\end{array}$ \\
\hline P10 & medium & $\begin{array}{c}\text { IT } \\
\text { architect }\end{array}$ & $\begin{array}{c}\text { IT service } \\
\text { provider }\end{array}$ \\
\hline P11 & medium & $\begin{array}{c}\text { IT } \\
\text { architect }\end{array}$ & $\begin{array}{c}\text { Public } \\
\text { transport }\end{array}$ \\
\hline
\end{tabular}

The interviews averaged at about 40 minutes, included a fixed set of questions and were recorded for later analysis. Five main question categories were discussed with the interview partners:

1. The maturity of EAM in the enterprise, to understand how the maturity of the EAM processes in an organization influence the understanding of EA planning

2. The interviewees' interpretation of EA planning

3. How EA planning is performed

4. How the EA planning process could be optimized

5. How optimal tool support would look like

Moreover, we transcribed the recorded interviews in order to be able to apply a coding procedure on them. For this purpose, the coding procedure of Corbin et al. [17] was applied using ATLAS.ti [37]. Hence, we broke down the data analytically and derived the first set of coded requirements. After that, we checked if there are overlapping requirements which we could merge into a new category. Finally, we derived the resulting requirement categories of the interviews which are shown in Table 1.

In the following, the answers to the interview questions are discussed in more detail and the requirements from Table 1 are highlighted.

Understanding EA planning. In this paragraph, we will discuss the above mentioned question 2 which is about the interviewees' interpretation of EA planning. For this purpose, the question was split into several subquestions including the motivation for planning, which planning processes are executed on which abstraction levels, what stakeholders are involved, and what the triggers for EA planning are.

Regarding the motivation for EA planning, the interviewees gave relatively similar answers. The most common opinion of those with an EAM background is that planning has the goal to develop several to-be architectures which are leading to a target architecture based on strategic decisions (four years and more in the future). On the other hand, in some organizations, EA planning additionally needs to be done for short-term (one to two years or less) decisions, like P6 mentioned. This can be due to fast regulatory changes or quality criteria which need to be complied to. Furthermore, the interviewees with a more technical background are interested in the technical relationships between the individual components and need to decide which technologies are outdated and which applications need to be shut down or updated.

During the interviews, many reasons for conducting EA planning were mentioned. The most often mentioned reason was consolidation, when e.g. systems are merged to avoid redundancies (P3, P4, P6, P9). But also migration planning, e.g. to change technologies 
(P4, P6) and to reduce costs (P1, P3) were mentioned. Another trigger was pending "make or buy" decisions, where the trade-offs between developing a solution inhouse or buying a specific software product are compared (P2). Additionally, the reliability and availability of systems (P1) and transformation planning (P3), where e.g. the management decides that a transformation is needed to be done, were mentioned. Furthermore, the identification of implementation projects (P5), reacting on legal requirements (P6), required decoupling of systems to reduce interdependencies (P6) were mentioned as triggers for EA planning. Also, conducting life-cycle management for information systems (P9) was mentioned. In more detail, if a core business application reaches the end of its support and a new solution needs to be deployed. An automated, tool-based trigger that notifies responsible employees of life-cycle changes was brought up as requirement in the expert interviews (R6). Further triggers are annual planning initiatives (P2, P3, P5, P11) and projects which have new requirements or which trigger a transformation themselves (P3, P6, P7, P9). Finally, establishing technological standardization (P10), creating a documentation for the change management (P8), and creating financial roadmaps (P11) were mentioned.

Regarding the abstraction level of the planning process, the opinions of the interviewees differed. This depended on the position of the interviewee in their organization but also on the individual opinions of the discussion partner. However, most of the interviewees agreed on the fact that EA planning starts on a high level of abstraction in order to discuss with decision makers. When the planning comes closer to the actual execution, the plans become more detailed. A high level of abstraction in EA planning means that only high-level concepts are discussed and implementation details are hidden. This is because the actual decisions are made by stakeholders who often do not have the time or expertise to make decisions based on highlydetailed plans. However, it is common that, once plans are approved, they proceed to a stage of more detailed planning that requires more detailed visualizations and integrates a different set of stakeholders.

The mentioned stakeholders of the EA planning initiative are mostly the budget owners, like IT-managers and other decision makers. When it comes to conducting the specific projects needed to fulfill the plan, the involved responsible IT architects and team leaders (P5) are also stakeholders. Depending on the business model, in some cases the requirements from the customers are included, like in the case of P1.

How EA planning is conducted. Each of the interviewees described a different planning process but in the essence a core Building-block are visualizations (R9) as the basis for discussion. In this context, the problem that the planning is based on an (often not upto-date) model of the current architecture (R1) was mentioned.

EAM tools are mostly used for capturing the current architecture. The actual planning and discussion of scenarios is mostly done by hand with the help of flipcharts, whiteboards, MS PowerPoint, and MS Visio. Participant P3 mentioned the use of reference data from their EAM tool for checking if their hand-crafted visualizations are valid.

Additionally, different scenarios are planned (R3) from which one is chosen to be implemented. Most of the interview partners had no specific process for the purpose of choosing the optimal option. Only few interviewees (P2, P5) based their decisions on predefined criteria or metrics, like monetary and technical criteria. Those who do perform this comparison do it mostly via Excel spreadsheets. In the rest of the cases, the decision on which scenario gets selected, is based on a visual comparison and discussion of the scenarios.

Optimization of the planning process. Nearly all of the interviewees had ideas of how their planning process could be optimized. One of the requirements for a better planning process was the usage of up-todate data and documentations (R11) which was mentioned by three interviewees (P3, P10, P11). According to P2 and P7, a software trigger, which warns when a specific software reaches the end of its life-cycle would enhance the timeliness of their planning activities. According to several participants, this knowledge is often available too late to set up transformation projects in a timely manner (R14).

Furthermore, the idea of a central repository (R16) was discussed. The advantage of such a repository is that all the needed information is consistent over all tools because it persists in only one database (P5, P10). Another problem which occurred is that EA planning ran out of scope (P9), i.e. it was conducted at too big or too small scale. Therefore, the according projects could not be sufficiently executed.

Moreover, interviewee P9 envisioned a virtual whiteboard for EA planning that mimics working on a physical whiteboard. The interviewee described it as a large touchscreen with which one is able to create architectural visualizations and automatically save them to an EA tool. The aim was to combine the group interaction characteristics of a white board for planning with the advantages of modeling with a software tool.

Optimal tool support. According to the question of how the optimal tool support would look like, the interviewees agreed on the need of visualizations for each stakeholder category (R9). This is especially important because, as mentioned above, different stakeholders need different levels of abstraction to comprehend a given problem. Additionally, the kind of visual- 
ization is important because, for strategic decision making, other visualizations are needed than for operational decision making. Another requirement is that it needs to be possible to easily generate visualizations from the as-is architecture (R12) and execute ad-hoc queries on these (P3). Also, visualizations need to be generated with a good performance and adequate default layout when large ones are generated (P5).

Another important aspect considering visualizations is that one needs to be able to freely paint on the visualization canvas (R13). This is important because most of the interviewees are conducting EA planning by hand right now, where drawing freely is not a problem. Hence, there needs to be a solution which simulates the freedom of a whiteboard or flipchart to freely annotate information and resemble the actual planning processes (P9, P11). Furthermore, there needs to be a possibility to plan multiple target models and compare them visually (R2, R3). The tool should also be able to export visualizations in a manner that they can be imported and visually enhanced in other drawing tools, and that they can be included in presentations.

A general tool requirement is that it has to be able to make heterogeneous data sources usable (R15). This is important because many enterprises have their data spread over several repositories and in different forms. Therefore, it is important to support the import of all required data sources to ensure completeness. Another tool requirement is that the tool generally needs to be easy to use (R12).

Summarizing, we tackled research question RQ1 by discussing the common understanding of EA planning among EA practitioners. Furthermore, we derived requirement categories as shown in Table 1 to cover our research question RQ2. Also, we answered research question RQ3 by discussing the different levels of abstraction on which plans are developed in practice, in the paragraph "Understanding EA planning”. Moreover, the interviews showed that practice faces challenges in the field of EA planning. In the following section the results of the structured literature review will be presented to show the current state of research on EA planning and to find out which requirements for EA planning can be derived out of literature.

\subsection{Structured Literature Review}

In this section, the structured literature review resulting in 32 publications on EA planning is presented. The goal of this review was to explore the current state of research on EA planning and to identify its mentioned and tackled requirements as well as the presented solutions.
The review was conducted based on the method of Armitage and Keeble-Allen [9]. The basis of their review method requires defining a research question. In our case these questions are the previously mentioned research questions RQ4 and RQ5:

"What is the current state of research on EA planning?" and "What requirements are identified and tackled in literature?"

After defining the research questions, a research protocol was created to predefine all relevant steps in the review process. It included the search terms, a timeline, inclusion criteria, exclusion criteria, the search strategy, as well as categories for the found literature.

As a first step, the search was conducted by using Google Scholar in combination with the defined search terms. For example, the keywords "Enterprise Architecture Planning" in combination with the term "Requirements" were used. For every search, the first 10 result pages were scanned, capturing title and abstract. Additionally, as proposed by Jalali and Wohlin [27], a backward search and a forward search on the resulting list of relevant papers was performed. The first was done via references and the second via Google Scholar's “citing papers" functionality. In addition to this, several books from practitioners were included in the review. The inclusion criteria for these books was the description of an EA planning process. Furthermore, due to the relatively small number of identified publications and the goal of getting a general overview, no exclusion criteria was defined for the research literature.

The review resulted in 32 relevant publications which are targeting solutions and requirements for EA planning. These publications were then categorized. Also, the identified requirements were coded and summed up over all relevant publications. The result of this coding procedure is shown in Table 1.

Most of the relevant literature was targeting the problem of evaluating EA models, the comparison of scenarios, and the transformation path for transforming the current architecture to a specific target architecture.

Furthermore, we identified two main clusters of researchers that published on the topic of EA planning. These are Aier et al. from the University of St. Gallen as well as Buckl et al. from the Technische Universität München. The group of Aier et al. focused on the process of transformation planning. For this purpose, they developed a process model for EA planning in [4]. Additionally, they modeled a system which accounts for the interplay of EA planning, requirements, release, and synchronization management and an integrated information model which describes EA transformation planning in [3]. Buckl et al. worked on an information model for application landscape evolution in [13]. Fur- 
thermore, they focused on how to model enterprise architecture transformations in [15]. The publications of the two groups were mostly of conceptual nature except for a prototype described in [14]. The evaluation of the publications was done via case studies.

In the following, the requirement categories shown in Table 1 are discussed in more detail. While Table 1 comprises the full list of references, in this section we summarize core ideas and concepts in an example which spans over all categories mentioned in the SLR.

R1) Plan based on current architecture. EA planning efforts usually start with the current architecture as the baseline for plans, as outlined by e.g. Javanbakht et al. [28] (R1b).

R2) Evaluate to-be and current architecture. As outlined by Jugel et al. [29], before the planning process is started, the current EA needs to be analyzed to find problems and possibilities for optimization (R1a, $\mathrm{R} 2 \mathrm{abc}$ ). This can be done with the help of key performance indicators (KPI). One common method for this purpose is to apply metrics to the current architecture and compare their values with the respective target value or optimum, like stated by Ahlemann [2] (R2c). Here, it can be distinguished between qualitative and quantitative metrics, where qualitative metrics highlight critical components and quantitative metrics show what needs to be restructured, as discussed by Postina et al. [39].

R3) Plan different scenarios. When EA planning is conducted, it results mostly in multiple representations of the targeted to-be architecture (R3a). Furthermore, this results in different possible transformation paths which lead from the current to the to-be architecture (R3b). Again, these strategic options need to be compared according to specific criteria like cost, implementation speed, or technical soundness (R2abc). For this purpose, e.g. impact analysis and graph comparison can be used (R2ab), as e.g. stated by Aier et al. [7]. For instance, with the help of the impact analysis, it is possible to predict the impact of a decision on the current architecture. The graph comparison helps to analyze the differences between the current and the tobe architecture.

R4) Set up transformation paths. According to Diefenthaler and Bauer [19], once a decision regarding the to-be architecture has been made, the respective transformation path needs to be analyzed in more detail. For this purpose, a transformation model, as mentioned by Aier and Gleichauf [5], is proposed (R4a). According to Cîmpan et al. [16] the information of how to get from one state to the next (transformation path) should not be captured in the considered model, but in an external construct. Additionally, this transformation model, containing the formalized transformation information, should be built before the trans- formation steps are applied, as outlined by Aier and Gleichauf [5]. According to Aier et al. [3], after the current and the desired to-be architecture are linked together, projects are derived from the to-be models (R4bcd). These projects are the driver of the transformation initiative.

R5) Define scope of plan. Each step in the transformation path is typically executed by a project. Therefore, as mentioned by Lautenbacher et al. [33], it is important to narrow the planning scope for determining the transformation path. Otherwise it could lead to a large amount of projects which cannot be conducted properly due to interdependencies, timing issues, or exploding costs (R5b).

According to Lautenbacher et al. [33], methods for deriving projects from to-be models and scope narrowing are for example gap analysis, segmentation analysis, and similarity measures (R4d). The gap analysis helps to derive projects from models by comparing the current and to-be model and finding gaps which need to be filled to ensure a successful transformation. Similarity measures and segmentation analysis can help to find problems which can be solved by conducting related projects and bundle them into work packages or into the same project (R4c).

R6) Manage architectural elements' life-cycle. In the next step, the derived projects need to be scheduled according to their temporal interdependencies and predecessor / successor relationships (R4b). Therefore, according to Buckl et al. [15], another important requirement for EA planning is to attach life-cycle information to each of the architectural elements like information systems (R6a). This is especially important for the aforementioned project schedule (R4b) and for finding temporal interdependencies, as mentioned by Aier and Gleichauf [5] (R6a).

R7) React on unplanned changes. According to Aier and Gleichauf [5], to-be architecture models need to be changed accordingly when their project schedule has unplanned changes or when changes on specific parts of the architecture are postponed. Therefore, the transformation model (R4a) should take dynamic changes into account (R7ab).

R8) Availability of an action repository. Furthermore, to check for consistencies in the transformation path and the planning process Diefenthaler et al. [18,19] and Lauterbach et al. [33] propose an action repository which consists of descriptions of possible changes in a way that allows the sequencing of actions. An abstract action which is part of this action repository consists of two parts. The first part specifies the preconditions for an action to be executable, while the second part specifies the changes to an architecture model if this abstract action is executed on it (R8ab). 
R9) Specific visualizations for EA planning. In the above-mentioned steps, usually different stakeholders are involved. According to Aier and Saat [8], the involvement of heterogeneous stakeholder groups may create conflicting requirements in a complex environment. Hence, an appropriate communication and documentation of the enterprise models is vital, as stated by Liles and Presley [35]. For this purpose, and, as mentioned by Pulkkinen [40], because different stakeholders are interested in different levels of abstraction and granularities, different visualization types are needed (R9ab). Examples for such visualizations are interactive dashboards or cockpit views for stakeholders who are interested in high-level information, like stated by Jugel and Schweda [30], and a network plan for those who are interested in low-level information, like mentioned by Aier and Gleichauf [6] (R9c).

R10) Capture historic information. Additionally, according to Aier et al. [7], the creation time of a plan should be captured (R6a) to ensure traceability of architecture decisions (R10a) and for the possibility of versioning artifacts (R10b). Furthermore, this timebased data should also be depicted in the visualizations.

R11) Availability of up-to-date data and documentation. The basis for all of the above-mentioned steps of EA planning is data. Therefore, according to Hanschke [22], it is important that up-to-date data and documentations with sufficiently high quality are available (R11ac).

R12) Availability of tool support. Aier et al. [5] propose the usage of a modeling tool in order to identify the necessary transformation steps and to establish a transformation procedure model. They also mention that such a tool must be able to compare and analyze models and propose a useful sequence of transformation steps which depends on the interdependencies and interrelations between the EA model elements.

In this latter section, the results of the literature review were presented.

We identified that the research in the field of EA planning is mostly focusing on EA evaluation, scenario planning, and building transformation paths. All the identified requirements are shown in Table 1. Only a small number of relevant literature was identified that is the output of 2 research groups and 18 authors. This could be reasoned by the fact that this research field is relatively new. Requirements like visualizations were mostly mentioned by the practitioners. This raises the question whether current research sufficiently covers the current needs of practitioners who are involved in EA planning activities. In the following section, the SLR results will be analyzed together with the interview results to elicit the gaps between the two.

\section{Analysis}

Table 1 depicts the requirements that are mentioned in literature and those that are mentioned in the interviews. Additionally, it can be seen that especially the requirement categories R4, R7, R8, and R10 are just mentioned in research literature which is an indicator that these requirements are currently more academic than practical. Also, the requirement categories R13, R14, R15, and R16 are solely mentioned by the interview participants which indicates that specific needs of practitioners are not addressed by current research.

Furthermore, as seen in the SLR, in research about EAM, visualizations are primarily used for depicting the current state of an architecture and to-be architectures on a fixed level of abstraction. On the other hand, in practice, visualizations on different levels of abstraction for different stakeholders are needed. These visualizations are further used for discussions and communication of the planning goals. Therefore, each stakeholder needs to receive the visualization based on their required level of abstraction to have a basis for discussion. Hence, the identified literature is not addressing the goal that visualizations are primarily a communication tool and therefore need to communicate the same information to different stakeholders on their specific abstraction level. Furthermore, we could not identify literature on visualizations which are specifically designed for the purpose of EA planning and comparing scenarios. According to this, visualizations for EA planning would be suitable for future research.

Other differences between practice and the research literature could be seen in the evaluation of EA models. In practice, the decision about different scenarios and plans are made primarily by visual comparison of the specific model, while the research literature proposes sophisticated methods for decision making. Based on our findings from the interviews, these methods appear impractical due to insufficient data quality. Also, during the interviews we found that the practitioners do not apply structured processes for evaluating scenarios as proposed by research. Therefore, there need to be simpler and more practical approaches to compare scenarios or to-be architectures. That is why more research is required in this direction.

Finally, a valuable research artifact would be a tool which is able to overcome the issues mentioned in the expert interviews, in combination with EA planning visualizations on different levels of abstraction, with good support for EA model comparison, and planning of multiple to-be architectures. 


\section{Future Work}

As a next step in establishing the current state of EA planning we will analyze the current landscape of commercial tools for EA planning. We will use the thereby gathered data to compare the features of the tools with the requirements from practice and the solutions proposed in the research literature.

Additionally, we will look at existing EA frameworks and evaluate their approaches to planning compared to our current findings.

Furthermore, as discussed in the previous section, a topic for future research is the development of a framework for EA planning visualizations. These should enable planning on multiple abstraction levels and should have tool support to connect them with the as-is architecture.

Also, the comparison and evaluation of multiple tobe architectures with each other or with the as-is architecture, is a topic which needs to be researched in more detail to have practical implications.

In order to evaluate our developed approaches and visualizations, we plan on implementing an EA planning tool, based on our previous work on automated EA documentation. This tool can then be evaluated by EAM experts in practice.

\section{Conclusion}

This paper presented the results from a series of expert interviews and a structured literature review on the topic of EA planning. One of the results of the expert interviews was that the interviewees had a different understanding of EA planning, as mentioned above. Another result was that the comparison and analysis of scenarios happened mostly visually in a non-structured way. Additionally, requirements for EA planning were derived and presented. Moreover, it could be seen that practitioners are struggling with challenges in the field of EA planning.

In the SLR it could be seen that the focus in the research on EA planning lies on EA evaluation, scenario planning, and building transformation paths. Also, just a small amount of relevant literature was found, which could be an indicator that progress in this field is driven by practice.

In our future work, we will address this issue by analyzing EA frameworks and commercial EA tools regarding EA planning. In addition, we will focus on developing a framework for EA planning visualizations and tooling that take the identified requirements into account.

\section{Acknowledgement}

This research was partially funded by the research projects txtureSA (FWF P 29022) and QE LaB - Living Models for Open Systems (FFG 822740).

\section{References}

[1] Agievich, V. and Skripkin, K. "Enterprise Architecture Migration Planning Using the Matrix of Change.” Proceedings of the Second International Conference on Information Technology and Quantitative Management (ITQM), (2014), pp. 231-235.

[2] Ahlemann, F., Stettiner, E., Messerschmidt, M., and Legner, C. Strategic Enterprise Architecture Management. Springer-Verlag, Berlin, Heidelberg, 2012.

[3] Aier, S., Buckl, S., Gleichauf, B., Matthes, F., Schweda, C.M., and Winter, R. "Towards a More Integrated EA Planning: Linking Transformation Planning with Evolutionary Change.” 5th International Workshop on Enterprise Modelling and Information Systems Architectures (EMISA), (2011), pp. 23-36.

[4] Aier, S. and Gleichauf, B. “Applying Design Research Artifacts for Building Design Research Artifacts: A Process Model for Enterprise Architecture Planning.” Global Perspectives on Design Science Research 6105, (2010), pp. 333-348.

[5] Aier, S. and Gleichauf, B. "Towards a Systematic Approach for Capturing Dynamic Transformation in Enterprise Models.” 43rd Hawaii International Conference on System Sciences, (2010), pp. 1-10.

[6] Aier, S. and Gleichauf, B. "Application of Enterprise Models for Engineering Enterprise Transformation.” Enterprise Modelling And Information Systems Architectures 1, 5 (2010), pp. 58-75.

[7] Aier, S., Gleichauf, B., Saat, J., and Winter, R. "Complexity Levels of Representing Dynamics in EA Planning." Proceedings of the 5th International Workshop on Cooperation \& Interoperability - Architecture \& Ontology (CIAO!), (2009), pp. 55-69.

[8] Aier, S. and Saat, J. "Understanding processes for modelbased enterprise transformation planning." International Journal of Internet and Enterprise Management 7, 1 (2011), pp. 84-103.

[9] Armitage, A. and Keeble-Allen, D. "Undertaking a Structured Literature Review or Structuring a Literature Review: Tales from the Field.” Electronic Journal of Business Research Methods 6, 2 (2008), pp. 103-114. [10] Bente, S., Bombosch, U., and Langade, S. Collaborative Enterprise Architecture - Enriching EA with Lean, Agile, and Enterprise 2.0 practices. Morgan Kaufmann, 2012. [11] Bernard, S.A. An Introduction to Enterprise Architecture: Third Edition. AuthorHouse, 2012. [12] Buckl, S., Ernst, A.M., Lankes, J., Matthes, F., and Schweda, C.M. "Enterprise Architecture Management Patterns - Exemplifying the Approach.” 12th International IEEE Enterprise Distributed Object Computing Conference (EDOC), (2008), pp. 393-402. 
[13] Buckl, S., Ernst, A.M., Matthes, F., and Schweda, C.M. "An Information Model for Managed Application Landscape Evolution.” Journal of Enterprise Architecture, (2009), pp. 12-26.

[14] Buckl, S., Ernst, A.M., Matthes, F., and Schweda, C.M. "Visual Roadmaps for Managed Enterprise Architecture Evolution." 10th ACIS Conference on Software Engineering, Artificial Intelligence, Networking and Parallel/Distributed Computing (SNPD), (2009), pp. 352-357.

[15] Buckl, S., Matthes, F., Monahov, I., and Schweda, C. "Modeling Enterprise Architecture Transformations." 3rd International IFIP WG5.8 Working Conference on Enterprise Interoperability (IWEI), (2011).

[16] Cîmpan, S., Verjus, H., and Alloui, I. "Dynamic Architecture Based Evolution of Enterprise Information Systems.” 9th International Conference on Enterprise Information Systems (ICEIS), (2007), pp. 221-229. [17] Corbin, J., Strauss, A., Clarke, A., Gerhardt, U., and Glaser, B. "Grounded Theory Research : Procedures, Canons and Evaluative Criteria.” Qualitative Sociology 13, 1 (1990), pp. 3-21.

[18] Diefenthaler, P. "Interactive Roadmap Generation in Enterprise Architecture Planning.” 3rd Enterprise Engineering Working Conference, (2013), pp. 1-14. [19] Diefenthaler, P. and Bauer, B. "From Gaps to Transformation Paths in Enterprise Architecture Planning.” In J. Hammoudi, Slimane; Cordeiro, José; Maciaszek, Leszek A.; Filipe, ed., Enterprise Information Systems. Springer International Publishing, 2013, pp. 474-489.

[20] Engels, G., Hess, A., Humm, B., et al. Quasar Enterprise - Anwendungslandschaften serviceorientiert gestalten. dpunkt.verlag, 2008.

[21] Ernst, A.M. and Schneider, A.W. "Roadmaps for Enterprise Architecture Evolution.” Workshop on Patterns in Enterprise Architecture Management (PEAM), (2010), pp. 253-266.

[22] Hanschke, I. Strategic IT Management: A Toolkit for

Enterprise Architecture Management. Springer-Verlag,

Berlin, Heidelberg, 2009.

[23] Hanschke, I. Lean IT-Management - einfach und effektiv. Hanser, 2014.

[24] Haren, V. TOGAF Version 9.1. Van Haren Publishing, 2011.

[25] Hevner, A.R. "A Three Cycle View of Design Science Research.” Scandinavian Journal of Information Systems 19, 2 (2007), pp. 87-92.

[26] Hevner, A.R., March, S.T., Park, J., and Ram, S.

"Design Science in Information Systems Research." MIS Quarterly 28, 1 (2004), pp. 75-105.

[27] Jalali, S. and Wohlin, C. "Systematic Literature Studies : Database Searches vs . Backward Snowballing." 6th ACM_IEEE International Symposium on Empirical Software Engineering and Measurement (ESEM), (2012), pp. 29-38. [28] Javanbakht, M., Rezaie, R., Shams, F., and Seyyedi, M. "A New Method for Decision Making and Planning in Enterprises.” 3rd International Conference on Information and Communication Technologies: From Theory to Applications (ICTTA), (2008), pp. 1-5.

[29] Jugel, D., Sandkuhl, K., and Zimmermann, A. “Towards Visual EAM Analytics: Explorative Research Study with
Master Students.” 7th International Workshop on Information Logistics and Knowledge Supply, (2014), pp. 13-21.

[30] Jugel, D. and Schweda, C.M. "Interactive Functions of a Cockpit for Enterprise Architecture Planning.” 18th International Enterprise Distributed Object Computing (EDOC), (2014), pp. 33-40.

[31] Keller, W. IT-Unternehmensarchitektur - Von der Geschäftsstrategie zur optimalen IT-Unterstützung. dpunkt.verlag, 2012.

[32] Lankhorst, M. Enterprise Architecture at Work Modelling, Communication and Analysis. Springer-Verlag, Berlin, Heidelberg, 2013.

[33] Lautenbacher, F., Diefenthaler, P., Langermeier, M., Mykhashchuk, M., and Bauer, B. "Planning Support for Enterprise Changes.” The Practice of Enterprise Modeling, vol. 165 of the series Lecture Notes in Business Information Processing, (2013), pp. 54-68.

[34] Leppänen, M., Valtonen, K., and Pulkkinen, M.

"Towards a Contingency Framework for Engineering an Enterprise Architecture Planning Method." 30th Information Systems Research Seminar in Scandinavia (IRIS), (2007), pp. $1-20$.

[35] Liles, D.H. and Presley, A.R. "Enterprise Modeling within an Enterprise Engineering Framework.” 28th Conference on Winter Simulation, (1996), pp. 993-999. [36] Luftman, J.N., Lewis, P.R., and Oldach, S.H. "Transforming the enterprise: The alignment of business and information technology strategies.” IBM Systems Journal 32, 1 (1993), pp. 198-221.

[37] Muhr, T. and Friese, S. User's Manual for ATLAS.ti 5.0. ATLAS.ti Scientific Software Development GmbH, Berlin, 2004.

[38] Niemann, K.D. Von der Unternehmensarchitektur zur IT-Governance. Vieweg+Teubner Verlag, 2005.

[39] Postina, M., Sechyn, I., and Steffens, U. "Gap analysis of application landscapes.” 13th IEEE International Enterprise Distributed Object Computing Conference Workshops (EDOCW), (2009), pp. 274-281.

[40] Pulkkinen, M. “Systemic Management of Architectural Decisions in Enterprise Architecture Planning. Four Dimensions and Three Abstraction Levels.” 39th Hawaii International Conference on System Sciences (HICSS), (2006), pp. 1-9.

[41] Pulkkinen, M. and Hirvonen, A. "EA Planning , Development and Management Process for Agile Enterprise Development.” 38th Annual Hawaii International Conference on System Sciences (HICSS), (2005), pp. 1-10. [42] Ramos, H. and Vasconcelos, A. "eXtreme Enterprise Architecture Planning.” 29th Annual ACM Symposium on Applied Computing (SAC), (2014), pp. 1417-1419.

[43] Saat, J., Aier, S., and Gleichauf, B. “Assessing the Complexity of Dynamics in Enterprise Architecture Planning - Lessons from Chaos Theory.” 15th Americas Conference On Information Systems (AMCIS), (2009), pp. 1-8. [44] Spewak, S. and Tiemann, M. "Updating the Enterprise Architecture Planning Model.” Journal of Enterprise Architecture 2, May (2006), pp. 11-19. 